\title{
UPPER JURASSIC AND LOWER CRETACEOUS SEDIMENTARY FORMATIONS FROM THE VÂLCAN MOUNTAINS (SOUTH CARPATHIANS)
}

\author{
GRIGORE POP \& IOAN I. BUCUR ${ }^{1}$
}

\begin{abstract}
The litho- and biostratigraphy of the Upper Jurassic-Lower Cretaceous formations cropping out in the southwestern part of Vâlcan Mountains is presented. Five formations were recognised in the studied area: Valea Pragurilor Formation, Valea Cheii Formation, Topeşti Formation, bioclastic-oolitic limestones from Sudoieşi valley and Izvarna Formation. The age of these formations is mainly based on foraminifera and calcareous algae.
\end{abstract}

Keywords: Lithostratigraphy, biostratigraphy, foraminifera, calcareous algae, Mesozoic, Vâlcan Mountains, South Carpathians.

\section{INTRODUCTION}

Except for some formations, the biostratigraphy and detailed lithology of the Jurassic and Cretaceous sedimentary deposits from the tectonic units of the outer Danubian Domain (outer Marginal Dacides, Săndulescu, 1984) of the South Carpathians, including the Vâlcan Mountains area, are generally poorly known. This is due to the scarcity of biostratigraphic markers within these deposits and their recrystallization caused by the late Senonian (Laramian) tectonics.

Our work focuses on the biostratigraphy and lithology of the Upper Jurassic and Lower Cretaceous formations outcropping in the southwestern part of Vâlcan Mountains. Our interpretations are based on the stratigraphic distribution of some micro-organisms, particularly foraminifera and calcareous algae, and include facies analyses performed on several sections and sequences.

\section{GEOLOGICAL SETTING}

The examined formations crop out in the outermost part of the southwestern area of Vâlcan Mountains and form a part of the alpine (Jurassic and Cretaceous) cover of the Laramian Mehedinți unit (Pop et al., 1988) or Lainici Nappe (Berza in Balintoni et al., 1989), largely extended in the outer Danubian Domain (Fig.1).

The studied succession includes the following lithostratigraphic entities (from bottom to top, Fig.2): a) siliciclastic (mainly arkosian) deposits of alluvial-lacustrine origin (lower part of the Lower Jurasic, Baia de Aramă Formation); b) transgressive marine mixed siliciclastic-carbonate rocks (probable middle to upper part of the Lower Jurassic); c) echinoid limestone (assigned to Middle Jurassic); d) calcarenitic skeletal and peloidal, often dolomitized limestones (Oxfordian, Valea Pragurilor Formation); e) regressive alluvial-lacustrine siliciclastic deposits (Upper OxfordianLower Kimmeridgian, Valea Cheii Formation); f) calcarenitic to calcilutitic limestones

${ }^{1}$ Babeş-Bolyai University, Department of Geology, M. Kogălniceanu str., 1, 3400 Cluj-Napoca, Romania. E-mail: ibucur@bioge.ubbcluj.ro 
frequently dolomitized (Kimmeridgian-Tithonian, Topeşti Formation); g) bioclasticoolitic limestone "formation" (cropping out in Sudoieş valley, Early Valanginian); h) urgonian type limestones (Barremian-Lower Aptian, Izvarna Formation), and i) the hemipelagites of Nadanova Formation (Upper Albian-Middle Turonian), capped by j) Senonian pro parte siliciclastic olistostrome, with olistoliths, reworked from older limestones, tectonically covered by the Severin and Getic nappes during the Laramian tectogenesis.

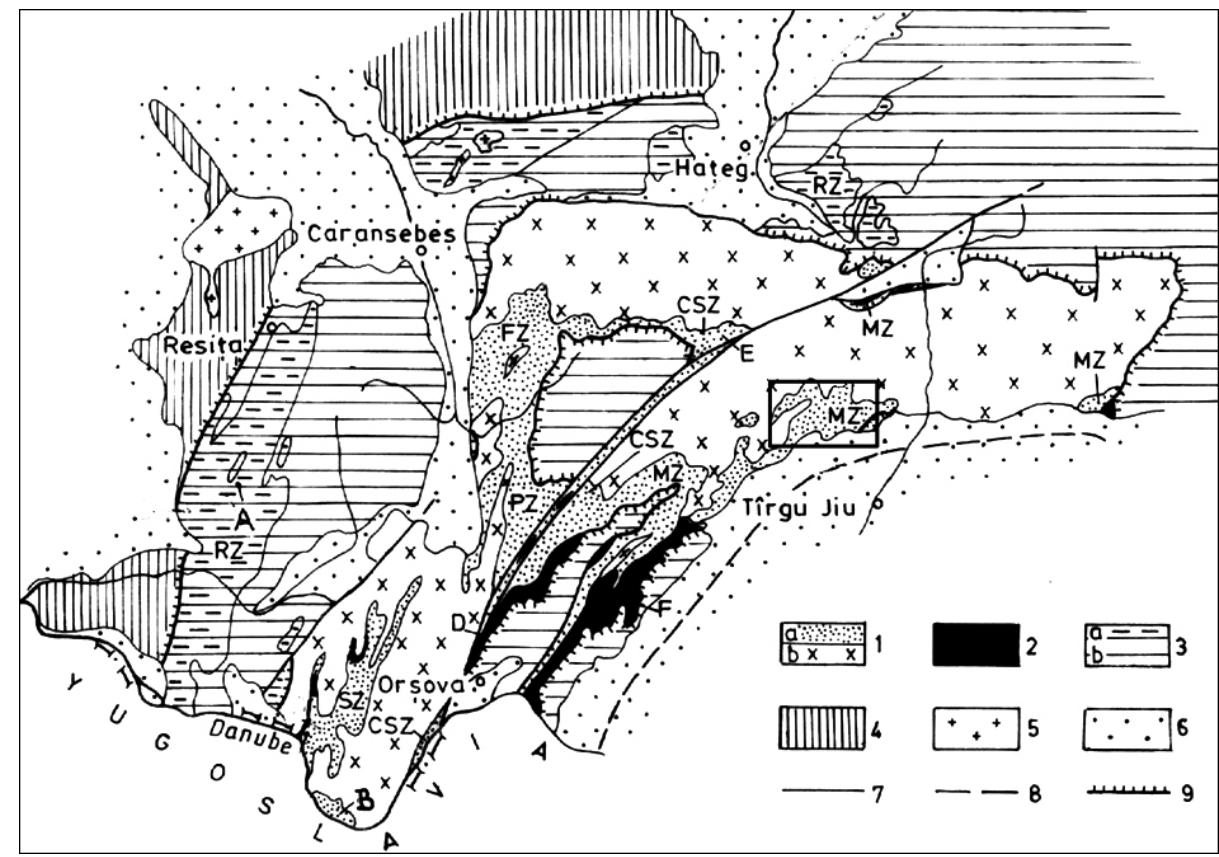

Figure 1 - Location of the studied area within the western part of the South Carpathians. 1 - Danubian domain (Marginal Dacides; a - Jurassic-Cretaceous sedimentary cover; b - crystalline basement and Paleozoic formations). 2 - Severin unit (External Dacides). 3 - Getic Nappe (Median Dacides; a - Mesozoic sedimentary cover; b - crystalline basement and pre-Jurassic sedimentary formations). 4 - Supragetic units (Median Dacides). 5 - Uppermost Cretaceous-Paleocene magmatites (Banatites). 6 - Tertiary sedimentary formations. 7 - fault. 8 - uncertain fault. 9 - overthrusting line. 10 - location of the studied area. RZ - Reşița zone; SZ - Şirinia zone; FZ - Feneş zone; PZ - Presacina zone; CSZ - Cazane-Stănuleț zone; MZ - Mehedinți zone.

The foregoing formations are bounded at their base by erosional unconformities, so they are more or less incompletely preserved.

The facies of the Jurassic and Lower Cretaceous formations illustrate a structural high during the corresponding time interval (Mehedinți threshold, Pop, 1973), which was gradually segmented by block-faulting linked to further extensional tectonics. 


\section{UPPER JURASSIC-LOWER CRETACEOUS FORMATIONS}

The investigated deposits are constituted of four shallow-marine carbonate formations, mainly representing the combined backstepping (BHST) and forestepping (FHST) system tracts, formed during some long-term eustatic cycles (second order), and a siliciclastic one. Successively they are (Fig.2):

1. Valea Pragurilor Formation (Oxfordian). It is a transgressive shallow-marine calcarenitic, often dolosparitic unit (<50 m thick), which consist of medium to thick bedded blackish and dark grayish limestones, showing four main microfacies:

- bioclastic-peloidal, sometimes sandy (basal part of the unit) packstone/ grainstone, including skeletal grains of echinoids, bivalves, gastropods, bryozoans, corals, foraminifera and algal crusts. Other associated grains are oncoids, micritized ooids and intraclasts. The carbonate grains, and even the quartz grains are coated by micritic envelopes and more or less micritized;

- ooidal-peloidal grainstone/packstone with bioclasts and rare oncoids;

- peloidal-bioclastic wackestone;

- peloidal mudstone.

Bioturbation and internal geopetal open spaces fillings were observed, especially in the uppermost part of this unit where the last two microfacies are dominant.

The microfacies and their vertical succession indicate an initial stage of marine deepening of BHST type, followed by a shallowing phase of FHST type.

2. Valea Cheii Formation (Uppermost Oxfordian-Lower Kimmeridgian). This formation, formerly defined by Pop $(1965,1973)$ and recently named Sohodol Formation by Stănoiu et al. (1997) ( a name already used in lithostratigraphy), represents a regressive siliciclastic unit (1-20 m thick) lying unconformably on the Valea Pragurilor Formation. It consists of alluvial-lacustrine quartzose, arkosian and lithic arenites and rudites, with reddish, greenish and greyish shale interbeds, locally thick and of red-bed type. Some thin intercalations of argillaceous micrites containing benthic foraminifera are also present (Fig.3).

The facies of these deposits, showing multiple vertical and lateral changes, suggests a widespread uplift and emergence of the area, associated with block-faulting and weathering of older geological formations (alpine cover and prealpine basement), generated by the late Jurassic tectonics.

The age of Valea Pragurilor and Valea Cheii formations is based on the following foraminiferal content: Alveosepta jaccardi (SCHRODT) (PI.II, fig.710; PI.III, fig.1-6), Parurgonina caelinensis CUVILLIER, FOURY \& PIGNATTIMORANO, ?Pseudocyclammina sp. and ?Neokilianina sp. Alveosepta jaccardi is an index species, of the Middle-Late Oxfordian-Early Kimmeridgian (Maync, 1960; Hottinger, 1967; Septfontaine, 1981; Pelissier \& Peybernes, 1982; Pelissier et al., 1984; Septfontaine et al., 1991; Tasli, 1993; Luperto-Sinni \& Masse, 1994; Bassoullet, 1997). 
GRIGORE POP \& IOAN I. BUCUR

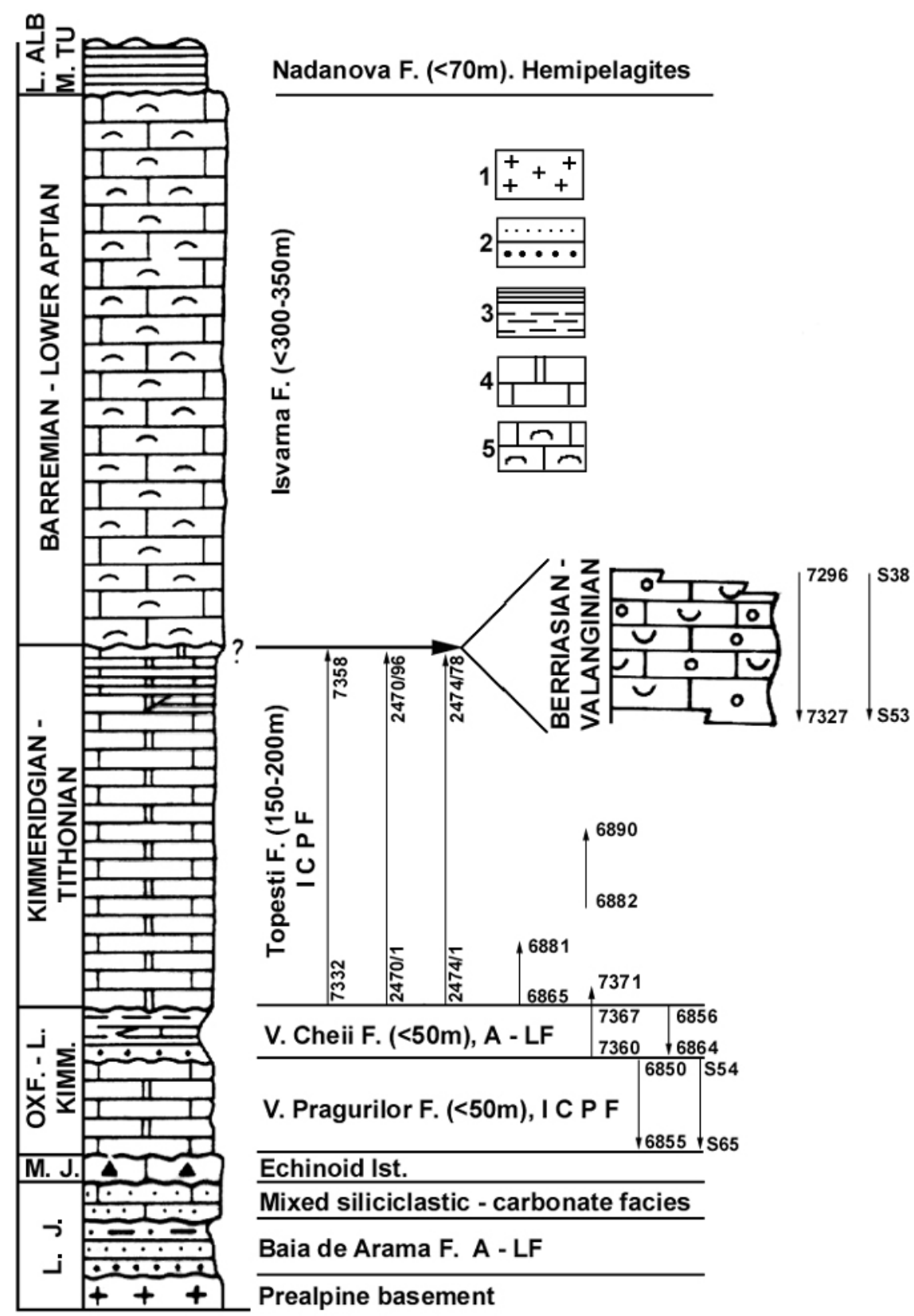

Figure 2 - Upper Jurassic and Lower Cretaceous deposits in the southwestern part of Vâlcan Mountains. A-LF - alluvial-lacustrine facies; ICPF - inner platform carbonate facies. 1-magmatic rocks; 2-conglomerates and sandstones; 3-shales; 4-limestones and dolomites; 5-Urgonian-type limestones; 6850-6855-sample numbers 


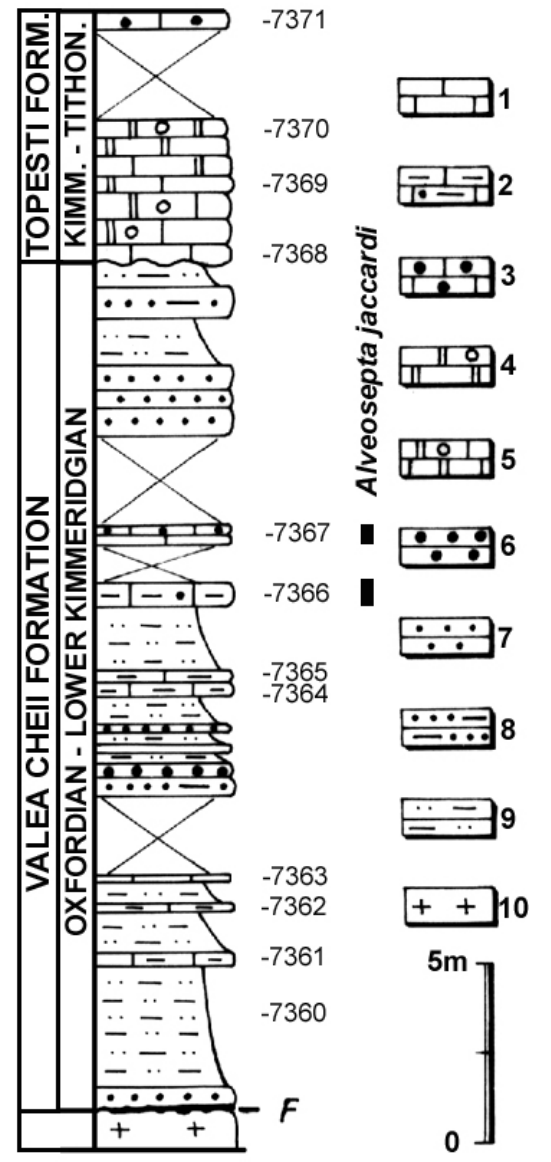

Figure 3 - Succession of the Valea Cheii Formation. 1-micritic limestones; 2-argillaceous limestones; 3-intraclastic limestones; 4-peloidal dolomites; 5-peloidal dolomitic limestones; 6-conglomerates; 7-sandstones; 8-shally sandstones; 9-sandy shales; 10-magmatic rocks.
3. Topeşti Formation (Kimmeridgian-Tithonian). This unit is also transgressive, lies unconformably on the Valea Cheii Formation, and consists of shallow-marine carbonates (150-200 m thick), which dominantly consists of thin to thick bedded, commonly blackish, often dolosparitic calcarenites and calcilutites, with different fabric. Its lower and middle parts are made up of the following microfacies in the order of their vertical frequency in the Sudoieş and south Sohodol sections: peloidal or peloidal-bioclastic wackestone and wackestone/ packstone, bioclastic-peloidal grainstone/ packstone, mudstone and peloidal musdstone/wackstone with rare ostracods and small foraminifera, and rare ooidal-intraclastic more or less bioclastic-peloidal grainstone / packstone (basal part). All these fabrics often include bioturbation, shallow intertidal to supratidal open-space (fenestral type) structures with internal sediment particularly of calcisiltite, followed by cimentation with blocky calcite. In some mudstone, calcisiltite microkracks infilling is present. In the upper part of the formation, the mudstone microfacies, more or less argillaceous, is dominant and represented by thin to medium bedded, blackish limestones, with parallel to subparallel laminae marked by a greater clay content or by small-sized dolosparite, open-spaces and microkracks with calcisiltite infillings. Other microfacies of wackestone/packstone and mudstone/wackstone type also occur. There are rare intercalations of brecciated, well sorted mudstone and peloidal grainstone interpreted here as tempestites.

An interesting micropaleontological assemblage was found in the limestones of Topeşti Formation, including: Megaporella boulangeri DELOFFRE \& BRUN (PI.II, fig.1-5), Salpingoporella annulata CAROZZI (PI.II, fig.6), Clypeina sulcata (ALTH) (PI.V, fig.3-5), Parurgonina caelinensis CUVILLIER, FOURY \& PIGNATTIMORANO (PI.III, fig.7, 8), Kurnubia palastiniensis HENSON (PI.III, fig.9), Neokilianina rahonensis (FOURY \& VINCENT) (PI.IV, fig.1, 2), Labyrinthina mirabilis 
WEYNSCHENK (PI.IV, fig.3-5, 8), Favreina cf. tabasensis BRÖNNIMANN (PI.V, fig.1, 2) and charphyte gyrogonites (PI.V, fig.6-9).

Megaporella boulangeri was described from the Kimmeridgian of Morocco (Deloffre \& Brun, 1986). The foraminiferal assemblage Parurgonina caelinensis, Kurnubia palastiniensis, Neokilianina rahonensis and Labyrinthina mirabilis is known from Upper Oxfordian-Tithonian time interval (Septfontaine et al., 1991; Bassoullet, 1997), but is mostly characteristic for the Kimmeridgian-Lower Tithonian.

4. A series of about $40 \mathrm{~m}$ of coarse grainstone with ferrous material in the matrix and grains, and with microsparitised grains crops out discontinously on Sudoieş valley. In the whole series the terrigenous quartz is present. Ooids, mositly micritised, or marginaly micritised peloids, dominate the grain fractions. Besides coral, bivalve, gastropod and echinoderm fragments, the samples collected from this formation contain also a very interesting assemblage of calcareous algae and foraminifera pointing to an early Cretaceous (Berriasian-Valanginian) age: Terquemella div. sp. (PI.VI, fig.13-17), Protopeneroplis banatica BUCUR (PI.VI, fig.1-9), Meandrospira favrei (CHAROLLAIS, BRÖNNIMANN \& ZANINETTI) (PI.VI, fig.10), Montsalevia salevensis (CHAROLLAIS, BRÖNNIMANN \& ZANINETTI), (PI.VI, fig.11), Feurtillia frequens MAYNC (PI.VI, fig.12), Andersenolina cherchiae (ARNAUD-VANNEAU, BOISSEAU \& DARSAC) (PI.VII, fig.1, 2), Andersenolina elongata (LEUPOLD) (PI.VII, fig.3, 7), Andersenolina delphinensis (ARNAUD-VANNEAU, BOISSEAU \& DARSAC) (PI.VII, fig.8, 10, 11), Andersenolina perconigi NEAGU (PI.VII, fig.9) and "Trocholina" molesta GORBATCHIK (PI.VII, fig.4, 5).

The assemblage is typical for Early Valanginian deposits in the Tethyan realm, and indicates a position of these limestones between the Topeşti and Izvarna formations.

5. Izvarna Formation (Barremian-Lower Aptian). The uppermost carbonate formation (250-350 $\mathrm{m}$ thick) is represented by urgonian limestones. It is commonly made of coarse bedded to massive grayish limestones. They consist of bedded or massive micrites, and include bedded bioclastic and peloidal grainstone to wackestone, containing miliolids, orbitolinids and rare rudists.

The age of Izvarna Formation is based on the following assemblage: Paleodictyoconus arabicus (HEN-SON) (PI.VIII, fig.1-3, 6), Neotrocholina friburgensis GUILLAUME \& REICHEL (PI.VIII, fig.4), ?Melathrokerion sp. (PI.VIII, fig.10), Neomeris cretacea STEINMANN (PI.VIII, fig.7), Salpingoporella exilis DRAGASTAN (PI.VIII, fig.8), Salpingoporella patruliusi BUCUR (PI.VIII, fig.9) and Terquemella sp. (PI.VIII, fig.5). Paleodictyoconus arabicus is an orbitolinid mainly restricted to the Late Barremian time interval (Schroeder \& Cherchi, 1979; Correia et al., 1982; Cherchi \& Schroeder, 1999).

\section{NOTE (loan I. Bucur)}

This paper was in preparation when the unexpected death of late Grigore Pop happened. I consider it necessary to publish the preliminary results of our common work without changing the Grigore Pop's concept on the stratigraphy of the region. 


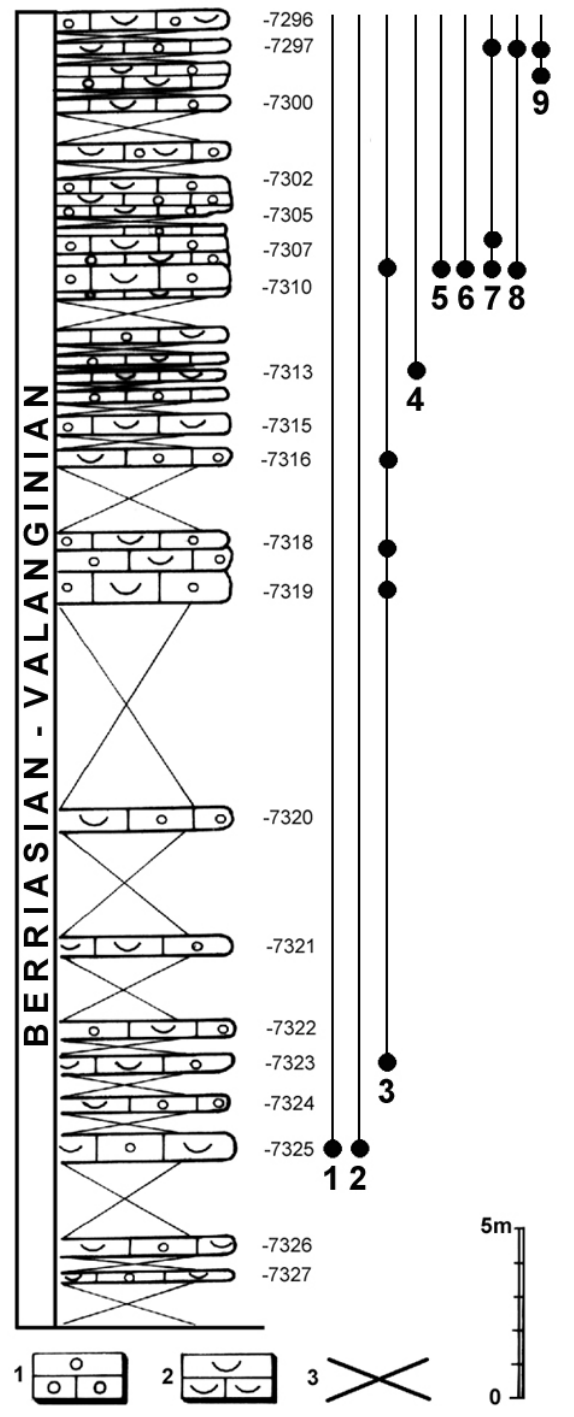

Figure 4 - Succession of the BerriasianValanginian bioclastic-oolitic grainstones from the Valea Sudoieş section. 1. Oolitic grainstone; 2-bioclastic grainstone; 3-covered interval. 1 - Feurtilia frequens; 2 - "Trocholina" molesta; 3 - Protopeneroplis banatica; 4 - Andersenolina perconigi; 5 - Meandrospira favrei; 6 Montsalevia salevensis; 7 - Andersenolina cherchiae; 8 - Andersenolina elongata; 9 Andersenolina delphinensis.
I must mention here that all the "formations" introduced in this paper are nomina nuda. The same mention should be made for the "formations" introduced by Stanoiu et al. (1997). All the names introduced by these authors are nomina nuda because the "formations" have no formal description (no designated stratotype, type locality, limits, lithological description, etc.). I made this remark to avoid further discussions about nomenclatural priority. In a written information (January, 2000) I. Stănoiu mentioned that the following "formations" described here were already separated and named as lithostratigraphic entities by Stănoiu et al. (1997):

"Izvarna Formation" (this paper) = "Tismana Member" (Stănoiu et al., 1997);

"Topeşti Formation" (this paper) = "Pocruia Member" and "Sodoieşu Member" (Stănoiu et al., 1997);

"Valea Cheii Formation" (this paper) = "Bâlta Member" of the "Sohodol Formation" (Stănoiu et al., 1997).

Without taking into account that all these "formations" are nomina nuda, the following remarks must be made:

1. Only "Izvarna Formation" (this paper) and "Tismana Member" (Stănoiu et al., 1997) have about the same age (Barremian-Aptian);

2. "Pocruia Member" and "Sodoieşu Member" were attributed by Stănoiu et al. (1997) to the Upper Berriasian-Hauterivian, while "Topeşti Formation" have a micropaleontological content allowing attribution to the Kimmeridgian-Tithonian. In turn, the bioclastic-oolitic limestones from Sudoieş valley (Fig.2, 4) described in this paper have a similar age with the one attributed to the "Pocruia Member" by Stănoiu et al. (1997) i.e. Early Valanginian, but a normal marine character and not a continental one (!?) 


\section{GRIGORE POP \& IOAN I. BUCUR}

3. The "Bâlta Member" of the "Sohodol Formation" is attributed to the Lower Cretaceous (Lower Berriasian) (Stănoiu et al., 1997), while the "Valea Cheii Formation" has definitely an Late Oxfordian-Early Kimmeridgian age indicated by Alveosepta jaccardi.

A simply conclusion arise from these comments: the Upper Jurassic-Lower Cretaceous deposits from the Cerna-Mehedinți-Vâlcan Mountains region are not enough studied. A real confusion exists at this time regarding the lithostratigraphic entities and their ages. I consider the present paper as a contribution to a better knowledge of the micropaleontological content and the age of some successions outcropping in well established sections in the southwestern part of Vâlcan Mountains.

\section{Acknowledgements}

I thank Mrs. Sivia Morariu for drawings and my collegue E. Săsăran for assistance in drawing and drafting the figures. I also thank J.P. Masse (Marseille) for the revision of the manuscript. This paper represents partially (loan I. Bucur) a contribution to the Project B24, grant ANSTI.

\section{REFERENCES}

Balintoni I., Berza T., Hann H.P., lancu V., Kräutner H.G., \& Udubaşa G. (1989) Precambrian metamorphics in the South Carpathians. Guide to excursion. 83 p., Inst. Geol. Geophys., Bucureşti.

Bassoullet J. P. (1997) - Foraminiféres. Les grands foraminiféres. In: Groupe français d'étude du Jurassique - Biostratigraphie du Jurassique ouest-européen et méditerranéen: zonations paralleles et distribution des invertébrés et microfossiles - Cariou, E. \& Haintzpergue P. (coord.) - Bull. Centre. Rech. Elf. Explor. Prod., Mém. 17, p.293-304, 3 tabl., Pau.

Cherchi, A. \& Schroeder, R. (1999) - Late Barremian orbitolinid foraminifera from northern Somalia. Bull. Soc. Paleontol. It., 38/1, p.3-13, Modena.

Correira, F., Berthou, P.-Y. \& Schroeder, R. (1982) - Découverte de Paleodictyoconus arabicus (Henson) foraminifère du Crétacé de l'Algarve oriental (Portugal). Implications stratigraphiques et paléobiogéographiques. C.R. Acad. Sc. Paris, 294, sér II, p.117119, Paris

Deloffre, R. \& Beun, N. (1986) - Megaporella boulangeri, nouvelle algue dasycladale du Kimmeridgien inférieur marocain. Revue de Micropaléontologie, 28/4, p.233-242, Paris.

Hottinger L. (1967) - Foraminifères imperforés du Mésozoique marocain. Notes et Memoires Serv. Geol., 209, 168 p., 61 fig., 20 pls., Rabat.

Luperto Sinni E. \& Masse J. P. (1994) - Precisazioni micropaleontologiche sulle formazioni di piattaforma carbonatica del Giurassico Superiore e del Cretaceo basale del massicio del Gargano (Italia Meridionale) e implicazioni stratigrafiche. Palaeopelagos, 4, p.243-266, 2 fig., 6 pls., Roma.

Maync, W.(1960) - Biocaractères et analyse morphométrique des espèces jurassiques du genre Pseudocyclammina (Foraminifères) II. Pseudocyclammina jaccardi (Schrodt). Revue de Micropaléontologie, 3/2, p.104-118, Paris.

Pelissié, T. \& Peybernès, P. (1982) - Etude micropaléontologique du Jurassique moyen/supérieur du Causse de Limogne (Quercy). Description des foraminifères Trocholina gigantea $n$. $s p$., Parinvolutina aquitanica n.gen., $n$. sp. et Limognella dufaurei $n$. gen., $n$. $s p$. Revue de Micropaléontologie, 25/2, p.111-132, Paris.

Pelissié B., Peybernès B. \& Rey J. (1984) - Les grands foraminifères benthiques du 
Jurassique moyen/supérieur du sud-ouest de la France (Aquitaine, Causses, Pyrénées). Intérêt biostratigraphique, paléoécologique et paléobiogéographique. In: Benthos '83, $2^{\text {nd }}$ int. Symp. Benthic Foraminifera (Pau, April, 1983), p.479-489, 5 fig., 2 pls., Pau.

Pop, G. (1965) - Prezența rocilor dolomitice în depozitele mezozoice din regiunea Tismana şi semnificația lor (Carpații Meridionali). St. cercet. Geol., geofiz., geogr., ser. Geologie, 10/1, p.23-37, Bucureşti.

Pop, G. (1973) - Depozitele mezozoice din Munții Vâlcan. Ed. Acad. RSR, 155 p., Bucureşti.

Săndulescu, M. (1984) - Geotectonica României. Ed. tehnică, 334 p., Bucureşti

Schroeder, R. \& Cherchi, A. (1979) - Upper Barremian-Lowermost Aptian orbitolinid foraminifers from the grand banks continental rise, nortwestern Atlantic (DSDP Leg 43, site 384). In: Tucholke, B.E. et al. (eds.) - Initial reports of the DSDP project, XLIII, p.575-583, Washington.

Septfontaine M. (1981) - Les foraminifères imperforés des milieux de plate-forme au Mésozoique: détermination pratique, interprétation phylogénétique et utilisation biostratigraphique. Revue de Micropaléontologie, 23 (3-4), p. 169-206, 10 fig., 3 pls., Paris.

Septfontaine M., Arnaud-Vanneau A., Bassoullet J.-P., Gusic I., Ramalho M. \& Velic I. (1991) Les foraminifères imperforés des plates-formes carbonatées jurassiques: état des connaissances et perspectives d'avenir. Bull. Soc. Vaud. Sc. Nat. (Bull. 312 Lab., Géol., Géophys. et Musée géol. Univ. Lausanne), 80 (3), p.255-277, 2 fig., Lausanne.

Stănoiu, I., Neagu, T., Dragastan, O., Melinte, M., Rădan, S. \& Baltres, A. (1997) - La stratigraphie des formations d'age Jurassique supérieur-Crétacé inférieur de l'unité de la Cerna dans la région du Plateau Mehedinți-Monts Vâlcan et Parâng (Carpathes Méridionales). St. cerc. Geologie, 42, p.63-80, Bucureşti.

Talsli, K. (1993) - Micropaléontologie, stratigraphie et environnement de dépôt des séries jurassiques à faciès de plate-forme de la région de Kale-Gümüshane (Pontides orientales, Turquie). Revue de Micropaléontologie, 36/1, p.45-65, Paris.

Plate I

\section{PLATES}

Fig.1-8, 11, 12 - Microfacies of the limestones from Topeşti Formation. 1-peloidal grainstone (sample 2474/14); 2-4, 12-peloidal-intraclastic grainstone (2-sample 2474/16; 3-sample 2474/22; 4-sample 2474/30; 12-sample7350); 5-sharp contact between a peloidal grainstone and a packstone/ wackestone (sample 2474/34); 6, 8-dolomitized mudstone/ packstone (6-sample 2474/63; 8-sample 2474/66); 7-bioclastic-peloidal grainstone (sample 2474/64); 11-laminitic-fenestral mudstone-packstone (sample7346).

Fig.9 - Microfacies of the limestones from the Berriasian-Valanginian deposits, Valea Sudoieş section. Bioclastic-oolitic grainstone (sample S51).

Fig.10 - Microfacies of the limestones from Valea Pragurilor Formation. Foraminiferal packstone (sample S54). All figs. X 10 (scale bar = 0,55 mm).

Plate II

Fig.1-5 - Megaporella boulangeri DELOFFRE \& BEUN. Sample 2474/49 (Topeşti Formation). 1-3, x 35; $4,5 \times 70$.

Fig.6 - Salpingoporella annulata CAROZZI. Sample 2470/72 (Topeşti Formation), x 50.

Fig.7-10 - Alveosepta jaccardi (SCHRODT). 7-sample S54 (Valea Pragurilor Formation), x 70; 810-sample S59 (Valea Pragurilor Formation); 8 x 35, 9-10 x 70.

Fig.11 - Parurgonina caelinensis CUVILLIER, FOURY \& PIGNATTI-MORANO. Sample S56 (Valea Pragurilor Formation), x 70. 


\section{GRIGORE POP \& IOAN I. BUCUR}

\section{Plate III}

Fig.1-6 - Alveosepta jaccardi (SCHRODT). 1, 5-sample 7367 (Valea Cheii Formation), x 70; 2, 3, 4sample 7361 (Valea Cheii Formation), x 70; 6-sample 7347B (Valea Cheii Formation), x 70.

Fig.7-8 - Parurgonina caelinensis CUVILLIER, FOURY \& PIGNATTI-MORANO. Sample 2474/8 (Topeşti Formation), x 35.

Fig.9 - Kurnubia palastiniensis HENSON. Sample 6871 (Topeşti Formation), x 70.

\section{Plate IV}

Fig.1-2 - Neokilianina rahonensis (FOURY \& VINCENT). 1-sample 6885 (Topeşti Formation), x 70; 2-sample 6853 (Topeşti Formation), x 70.

Fig.3-5, 8 - Labyrinthina mirabilis WEYNSCHENK. Sample 6885B (Topeşti Formation), x 70

Fig.6, 7, 9 - Mohlerina basiliensis (MOHLER).. 6-sample 7309D, x 70; 7-sample 7326B, x 70; 9sample $7317 \mathrm{~b}, \times 70$. Berriasian-Valanginian grainstones from Sudoieş valley section.

Plate V

Fig.1, 2 - Favreina cf. tabasensis BRÖNNIMANN. 1-sample 2470/53 (Topeşti Formation), x 70; 2sample 2470/60 (Topeşti Formation), x 70.

Fig.3-5 - Clypeina sulcata (ALTH). 3, 5-sample 2474/14 (Topeşti Fornation), x 35; 4-sample 2470/51 (Topeşti Formation), x 35.

Fig.6-9 - Charophytes gyrogonites. Sample 2470/61 (Topeşti Formatiopn), x 70.

\section{Plate VI}

Fig.1-9 - Protopeneroplis banatica BUCUR. 1, 3, 4-sample 7308A, x 140; 2-sample 7326b, x 140; 5 , 7-sample 7316, x 140; 6-sample 7319b, x 140; 8-sample 7308A, x 70; 9-sample 7322, x 70.

Fig.10 - ? Meandrospira favrei (CHAROLLAIS, BRÖNNIMANN \& ZANINETTI). Sample 7308A, x 140.

Fig.11 - Montsalevia salevensis (CHAROLLAIS, BRÖNNIMANN \& ZANINETTI). Sample 7308A, x 140.

Fig. 12 - Feurtilia frequens MAYNC. Sample 7326, x 140.

Fig.13-17 - Terquemella div. sp. 13-sample 7322, x 70; 14-sam[ple 7314C, x 70; 15-sample 7321b, x 70; 16-sample 7317, x 70; 17-sample 7315A, x70.

All samples from the Berriasian-Valanginian grainstones of Sudoieş valley section.

Plate VII

Fig.1, 2 - Andersenolina cherchiae (ARNAUD-VANNEAU, BOISSEAU \& DARSAC). 1-sample 7308A, x 70; 2-sample 7307, x 70 .

Fig. 3, 7 - Andersenolina elogata (LEUPOLD). 3-sample 7308C, x 35; 7-sample 7297C, x 35.

Fig.4, 5 - "Trocholina" molesta GORBATCHIK. 4-sample 7326b, x 70; 5-sample 7326C, x 70.

Fig.6, 8, 10, 11 - Andersenolina delphinensis (ARNAUD-VANNEAU, BOISSEAU \& DARSAC). 6sample 7297A, x 70; 8-sample 7299B, x 70; 10, 11-sample 7299, x 35.

Fig.9 - Andersenolina perconigi NEAGU. Sample 7313B, x 70.

All samples from the Berriasian-Valanginian grainstones of Sudoieş valley section.

\section{Plate VIII}

Fig.1-3, 6 - Paleodictyoconus arabicus (HENSON). 1-sample 100C (Izvarna Formation), x 35; 2-sample 100E (Izvarna Formation), x 70; 3-sample 100B (Izvarna Formation), x 70.

Fig.4 - Neotrocholina fribourgensis GUILLAUME \& REICHEL. Sample 100C (Izvarna Formation), $\mathrm{x} 70$.

Fig.5 - Terquemella sp. Sample 100B (Izvarna Formation), x 70.

Fig.7 - Neomeris cretacea STEINMANN. Sample 100B (Izvarna Formation), x 70.

Fig.8 - Salpingoporella exilis (DRAGASTAN). Sample 100C (Izvarna Formation), x 35.

Fig.9 - Salpingoporella patruliusi BUCUR. Sample 100E (Izvarna Formation), x 35.

Fig.10 - ?Melathrokerion sp. Sample 100E (Izvarna Formation), x 35. 


\section{PLATE I}
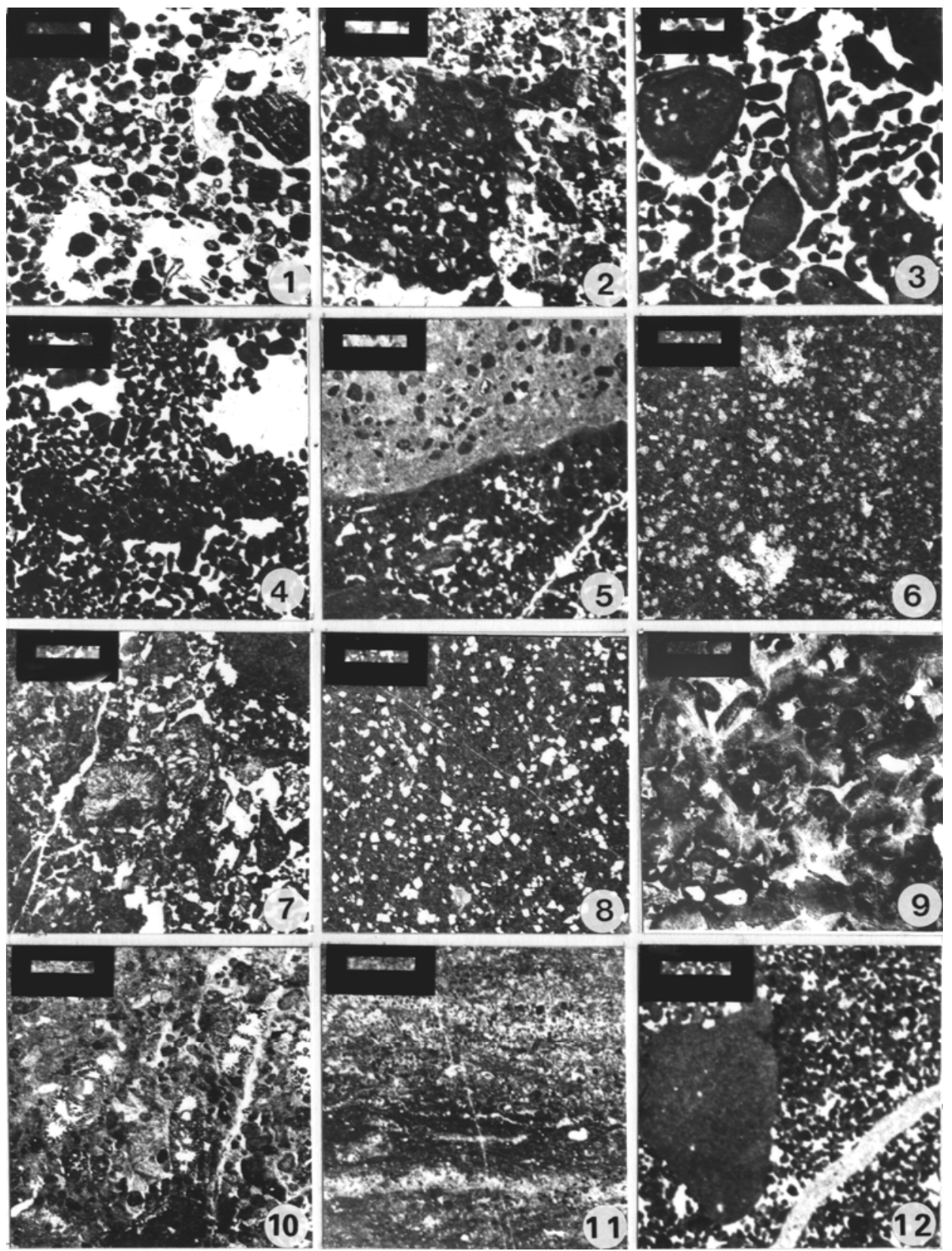
GRIGORE POP \& IOAN I. BUCUR

PLATE II
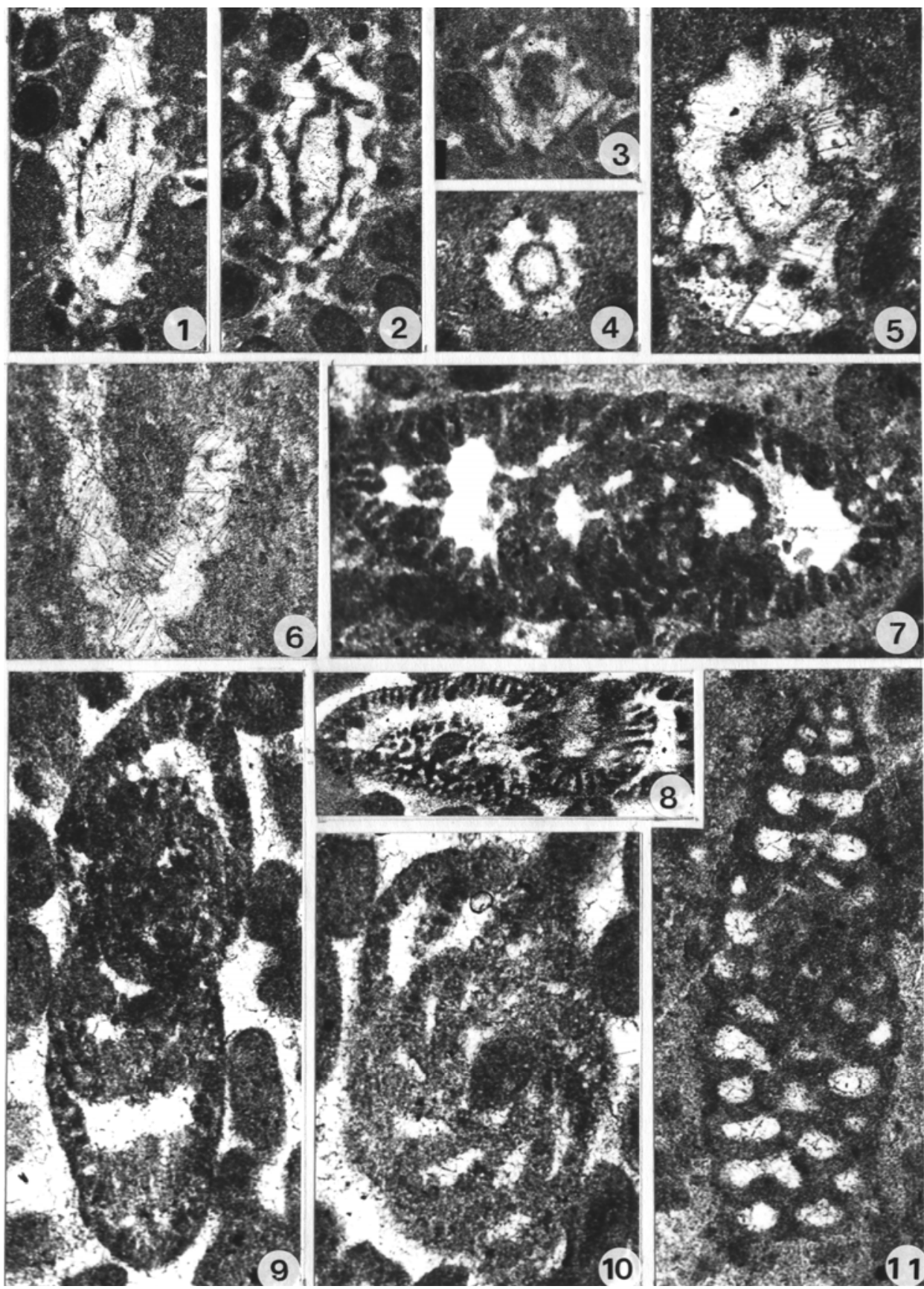


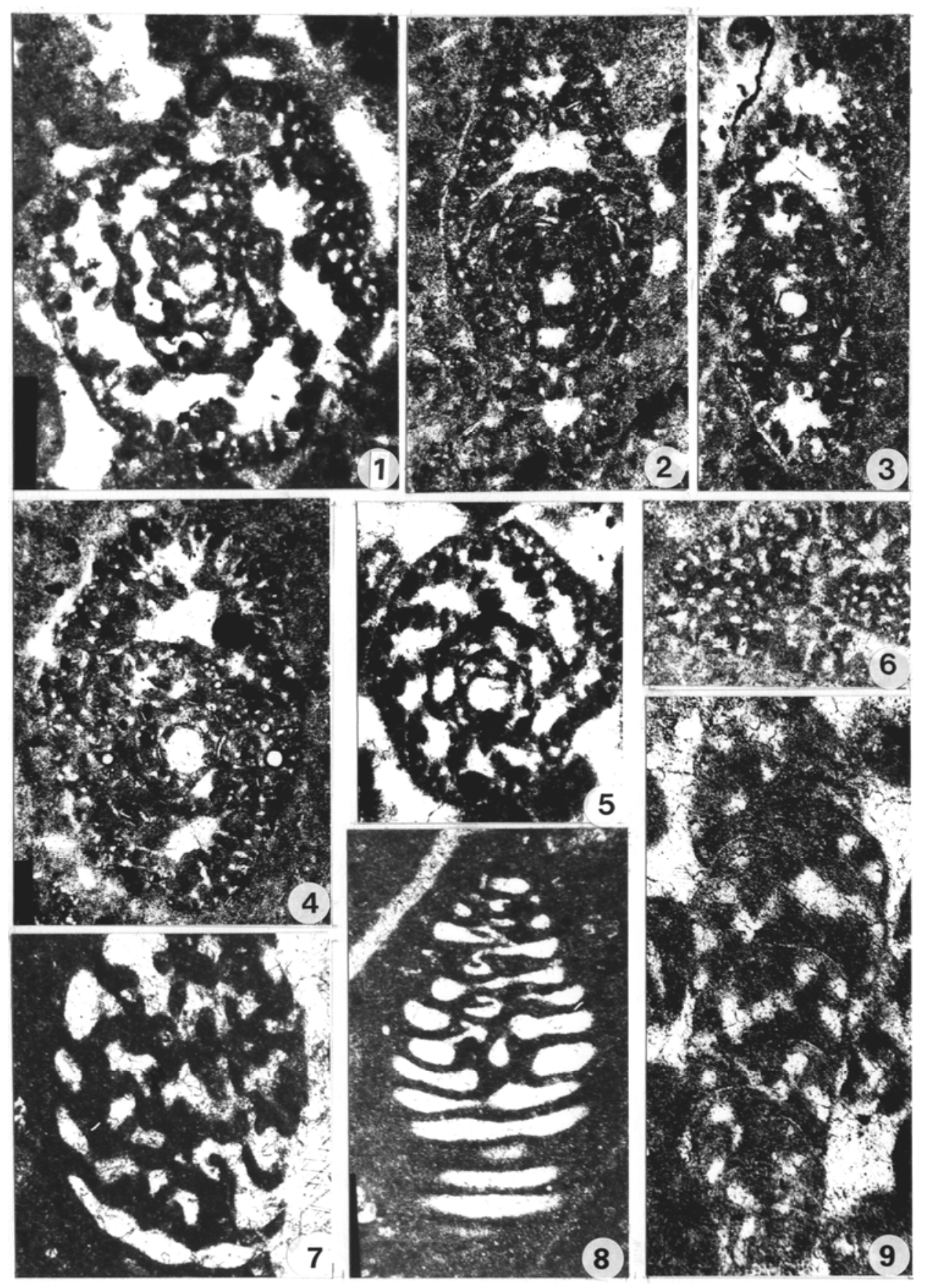


GRIGORE POP \& IOAN I. BUCUR

PLATE IV
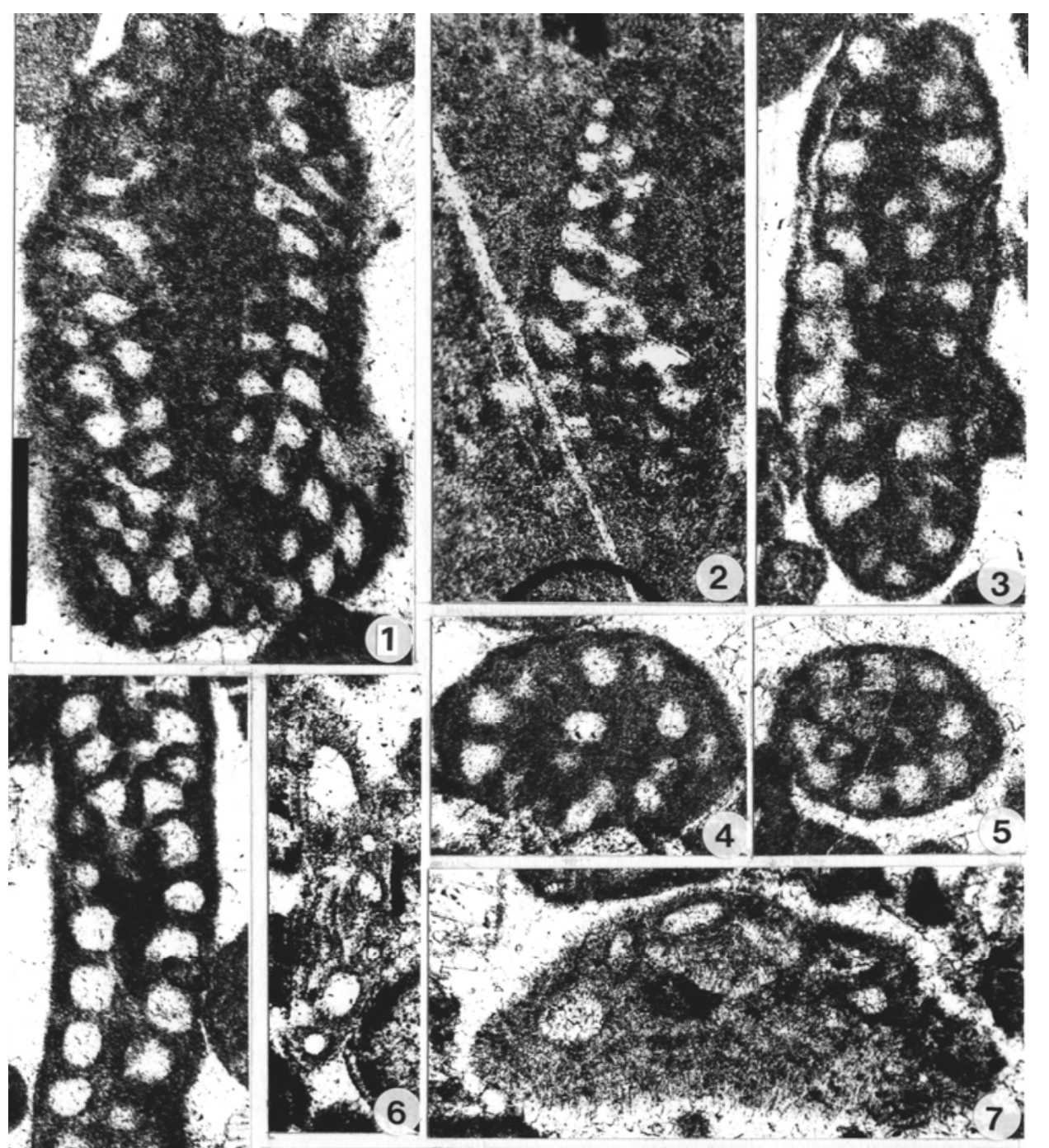

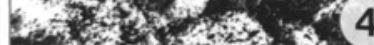
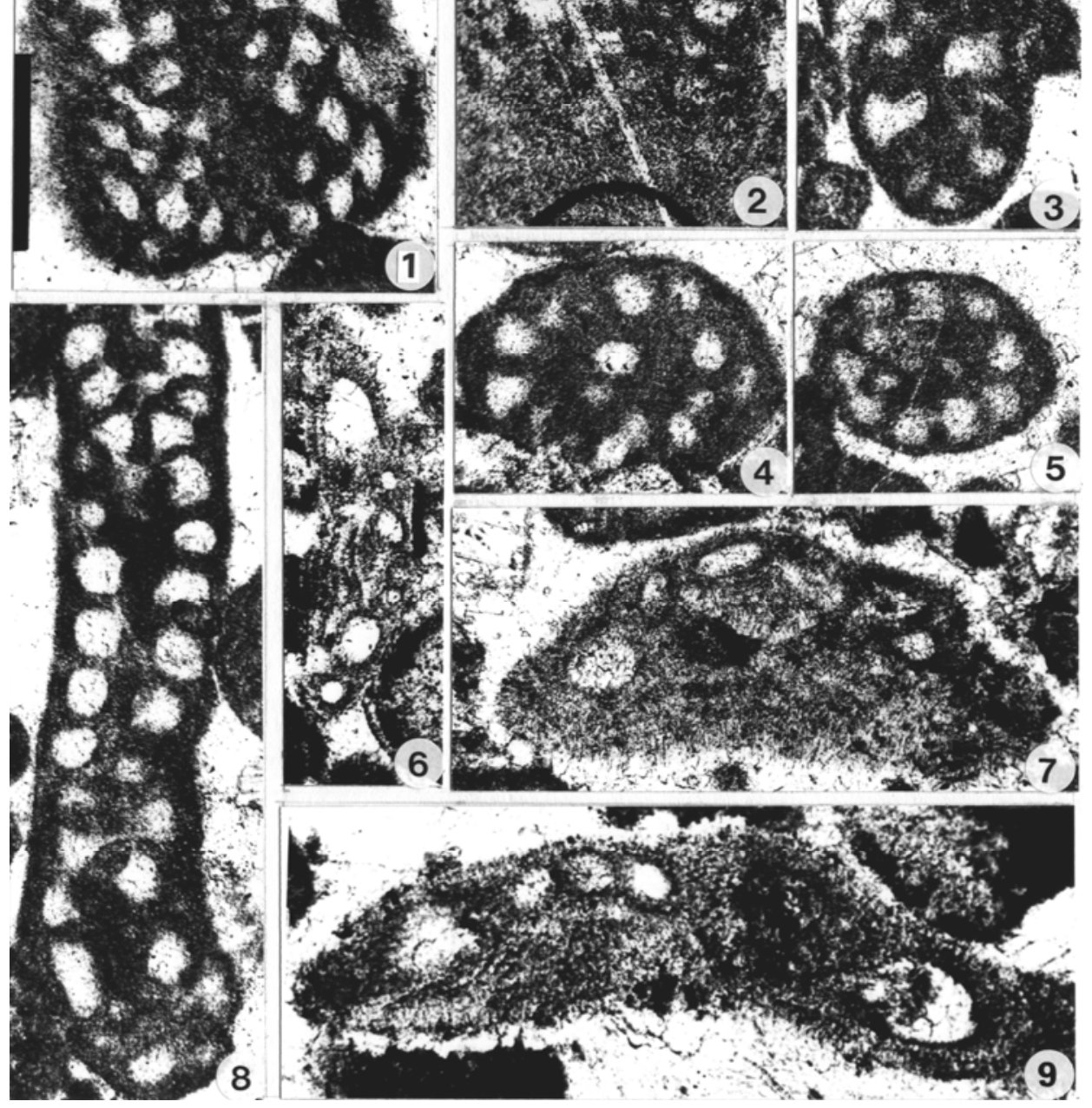
PLATE V
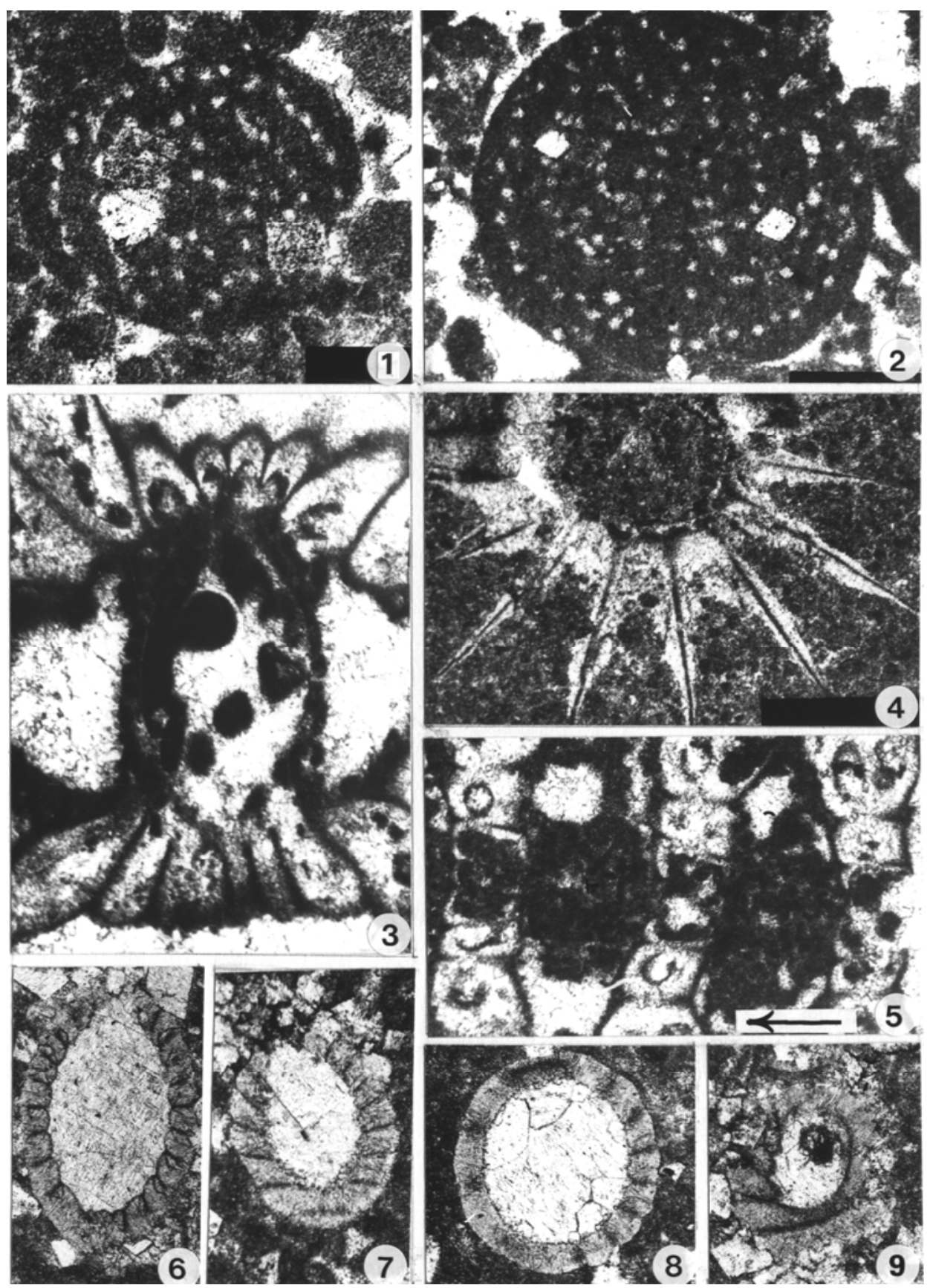
GRIGORE POP \& IOAN I. BUCUR

PLATE V

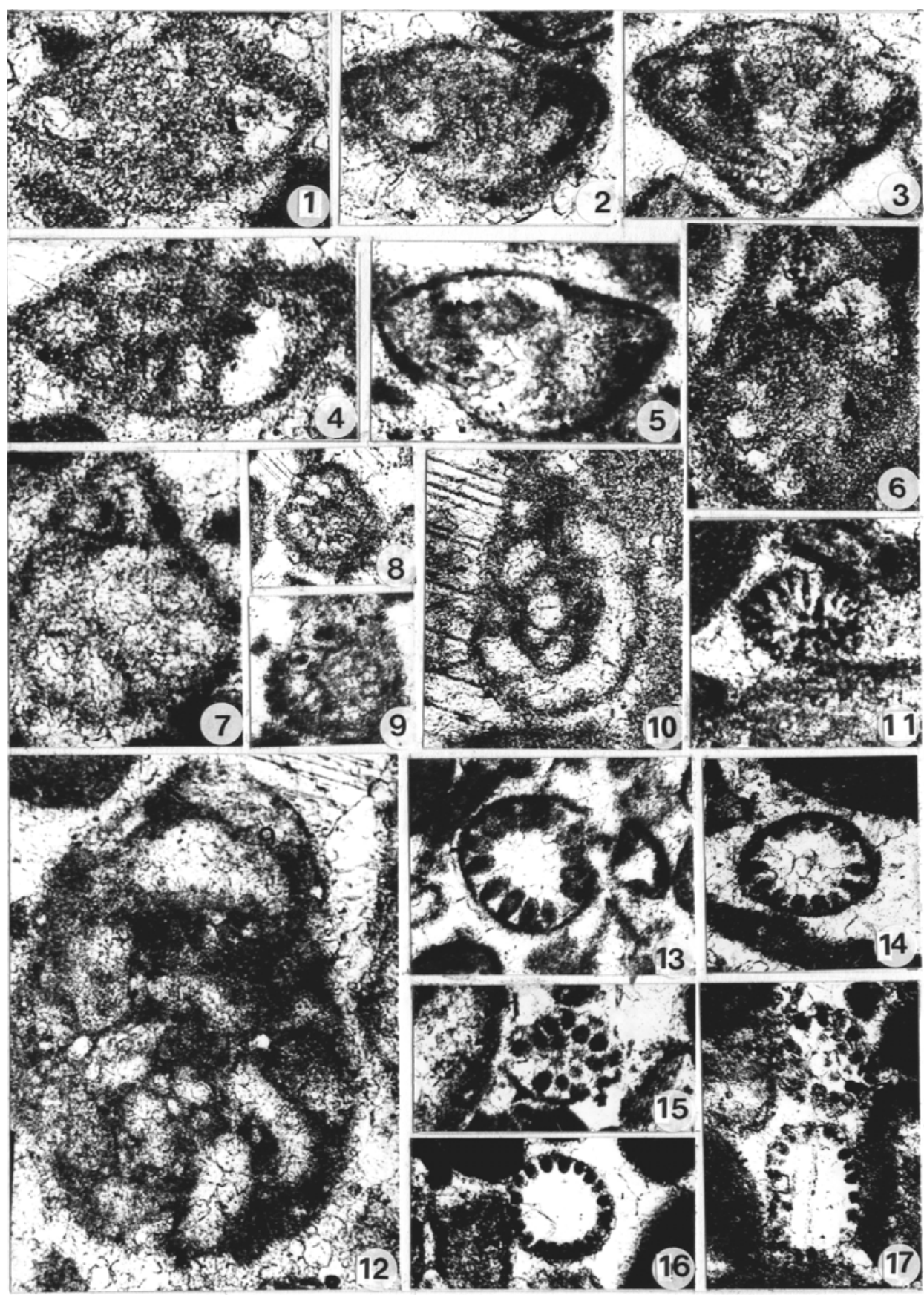


PLATE VII

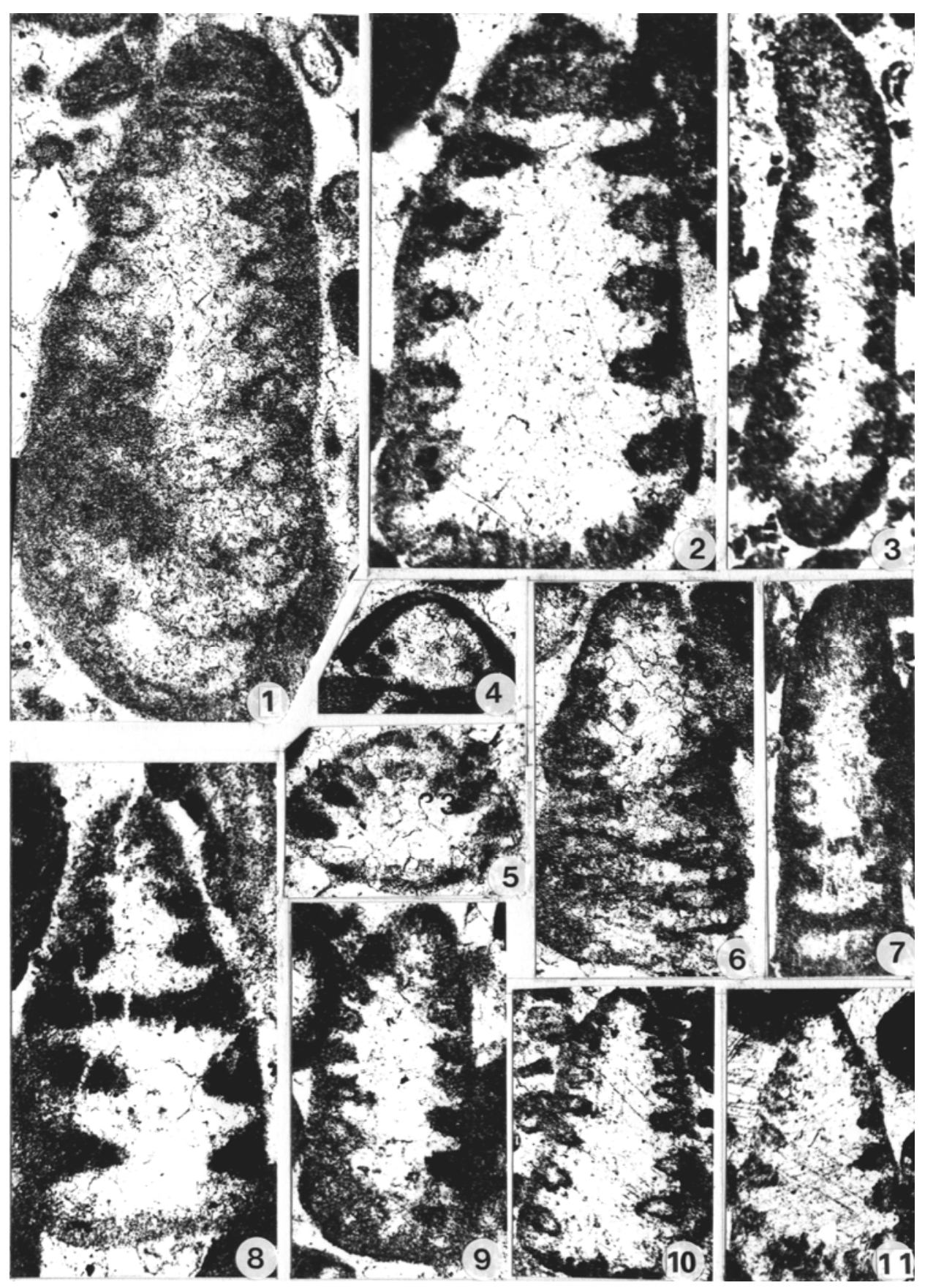


GRIGORE POP \& IOAN I. BUCUR

PLATE VIII
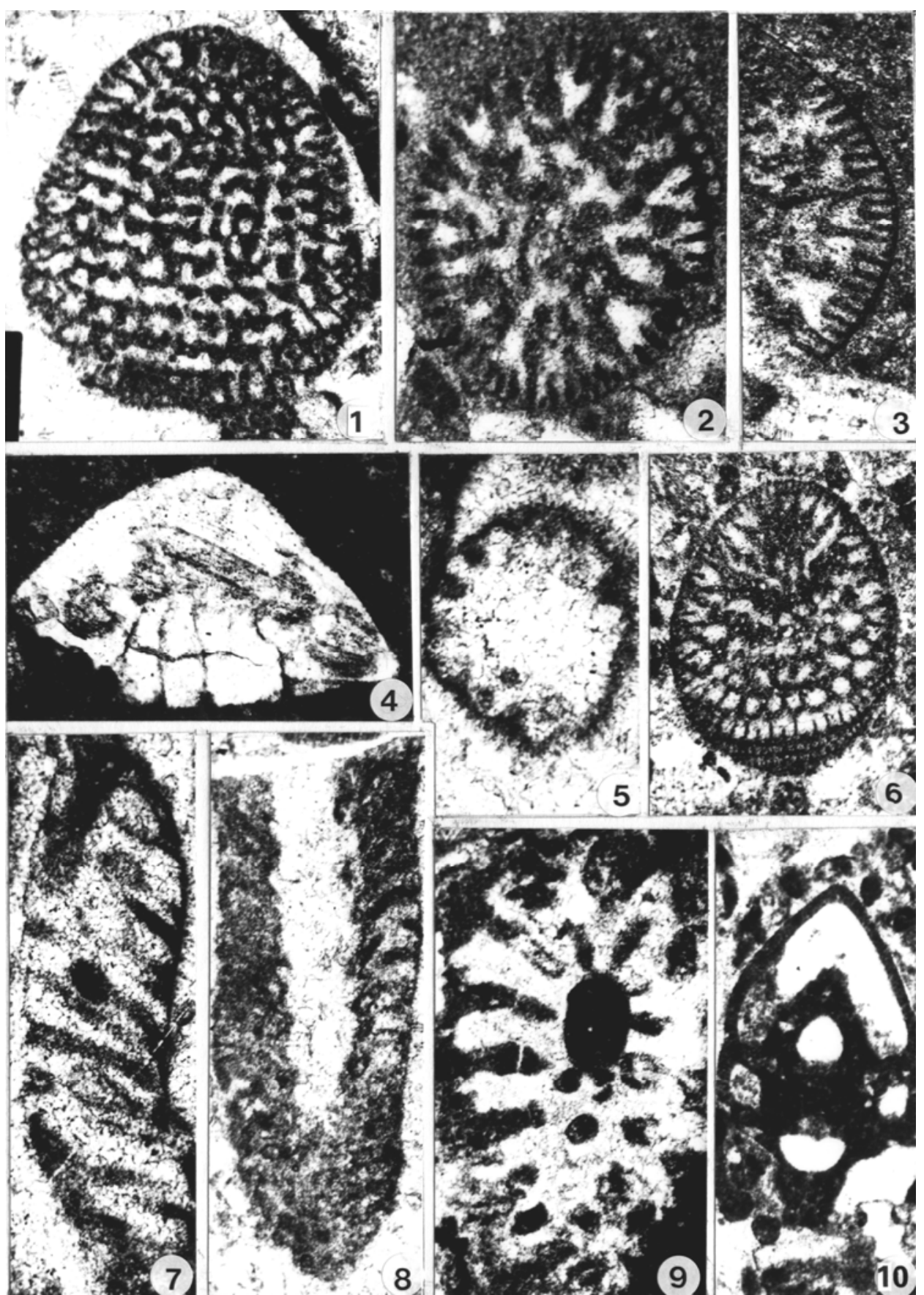

sid 